\title{
Magnetic order in the quasi-one-dimensional spin-1/2 molecular chain compound copper pyrazine dinitrate
}

\author{
T. Lancaster,* S. J. Blundell, M. L. Brooks, and P. J. Baker \\ Clarendon Laboratory, Department of Physics, Oxford University, Parks Road, Oxford, OX1 3PU, United Kingdom \\ F. L. Pratt \\ ISIS Facility, Rutherford Appleton Laboratory, Chilton, Oxfordshire OX11 0QX, United Kingdom \\ J. L. Manson \\ Department of Chemistry and Biochemistry, Eastern Washington University, Cheney, Washington 99004, USA \\ C. P. Landee \\ Department of Physics, Clark University, Worcester, Massachusetts 01610, USA \\ C. Baines \\ Swiss Muon Source, Paul Scherrer Institut, CH-5253, PSI Villigen, Switzerland
}

(Received 25 October 2005; published 27 January 2006)

\begin{abstract}
We present evidence of magnetic order in the quasi-one-dimensional spin-1/2 molecular chain compound, copper pyrazine dinitrate $\mathrm{Cu}\left(\mathrm{C}_{4} \mathrm{H}_{4} \mathrm{~N}_{2}\right)\left(\mathrm{NO}_{3}\right)_{2}$. Zero field muon-spin relaxation measurements made at dilution refrigerator temperatures show oscillations in the measured asymmetry characteristic of a quasistatic magnetic field at the muon sites. Our measurements provide convincing evidence for long-range magnetic order below a temperature $T_{\mathrm{N}}=107(1) \mathrm{mK}$. This leads to an estimate of the interchain coupling constant of $\left|J^{\prime}\right| / k_{\mathrm{B}}$ $=0.046 \mathrm{~K}$ and to a ratio of $\left|J^{\prime} / J\right|=4.4 \times 10^{-3}$.

DOI: 10.1103/PhysRevB.73.020410

PACS number(s): 75.10.Pq, 75.50.Ee, 76.75.+i
\end{abstract}

Experimental studies of quantum many-body physics rely on the existence of simple and well characterized model systems. One such system is the linear chain Heisenberg antiferromagnet (LCHAFM) which is described by the Hamiltonian

$$
H=\sum_{i}\left[-J \mathbf{S}_{i} \mathbf{S}_{i+1}+g \mu_{\mathrm{B}} \mathbf{B} \cdot \mathbf{S}_{i}\right]
$$

where $J$ is the nearest neighbor exchange constant along the chain and $\mathbf{B}$ is an applied magnetic field. For $\mathbf{B}=0$, the system will not show long-range magnetic order (LRO) for $T>0$, but will display excitations in the form of $S=1 / 2$ spinons which form a gapless, two-particle continuum. ${ }^{1}$ The magnetic field $\mathbf{B}$ can tune the system through a quantum critical point $(\mathrm{QCP}){ }^{2}$ (i.e., a zero-temperature phase transition) at $|\mathbf{B}|=\left|2 J / g \mu_{\mathrm{B}}\right|$ where the spin state becomes fully polarized. ${ }^{3}$

Experimental realizations of the LCHAFM have been made in several $\mathrm{Cu}$-chain compounds, the most successful of which is copper pyrazine dinitrate $\mathrm{Cu}\left(\mathrm{C}_{4} \mathrm{H}_{4} \mathrm{~N}_{2}\right)\left(\mathrm{NO}_{3}\right)_{2}$ or CuPzN. This material has a relatively low value of $J$ $=10.3(1) \mathrm{K}$, determined from high field magnetization and specific heat measurements, ${ }^{4}$ ensuring that the QCP is at an experimentally accessible magnetic field, but also a small ratio of intrachain to interchain exchange constants (estimated as ${ }^{5}\left|J^{\prime} / J\right|<10^{-4}$ ), showing that the chains are well isolated. This has allowed the magnetic field dependence of the excitations in the two-spinon continuum to be measured and has led to the identification of an extended critical state ${ }^{6}$ confirming long-standing predictions of theoretical and numerical studies. ${ }^{7}$ The mapping of $\mathrm{CuPzN}$ onto a onedimensional (1D) model is possible only if the chains are adequately separated. Three-dimensional (3D) LRO has not been detected in CuPzN down to $70 \mathrm{mK}$ on the basis of specific heat measurements. ${ }^{8}$ However, as we shall demonstrate, implanted positive muons are a much more sensitive probe of 3D LRO than specific heat for the case of very anisotropic magnetic chains. In this paper we show that CuPzN does indeed undergo a transition into a state of 3D LRO and we provide an estimate of $\left|J^{\prime} / J\right|$ which quantifies the degree of magnetic isolation of the chains and which is considerably larger than previously believed.

Zero-field muon-spin relaxation (ZF $\mu^{+} \mathrm{SR}$ ) measurements ${ }^{9}$ were made on a polycrystalline sample of CuPzN using the LTF instrument at the Swiss Muon Source $(\mathrm{S} \mu \mathrm{S})$, Paul Scherrer Institut, Villigen, Switzerland and the MuSR instrument at the ISIS facility, Rutherford Appleton Laboratory, UK. The sample took the form of as-grown crystallites, randomly oriented and attached to a silver plate using silver paint. This ensured good thermal contact and minimized any background depolarizing signal (silver has no nuclear moment). In a $\mu^{+} \mathrm{SR}$ experiment ${ }^{9}$ spin-polarized muons are implanted into the sample. The quantity of interest is the asymmetry $A(t)$, which is proportional to the spin polarization of the muon ensemble.

Examples of $\mathrm{ZF} \mu^{+} \mathrm{SR}$ spectra measured using a dilution refrigerator at the $\mathrm{S} \mu \mathrm{S}$ are shown in Fig. 1. In spectra measured below a temperature $T=110 \mathrm{mK}$ oscillations in the measured asymmetry are observed [Fig. 1(a)]. These oscilla- 


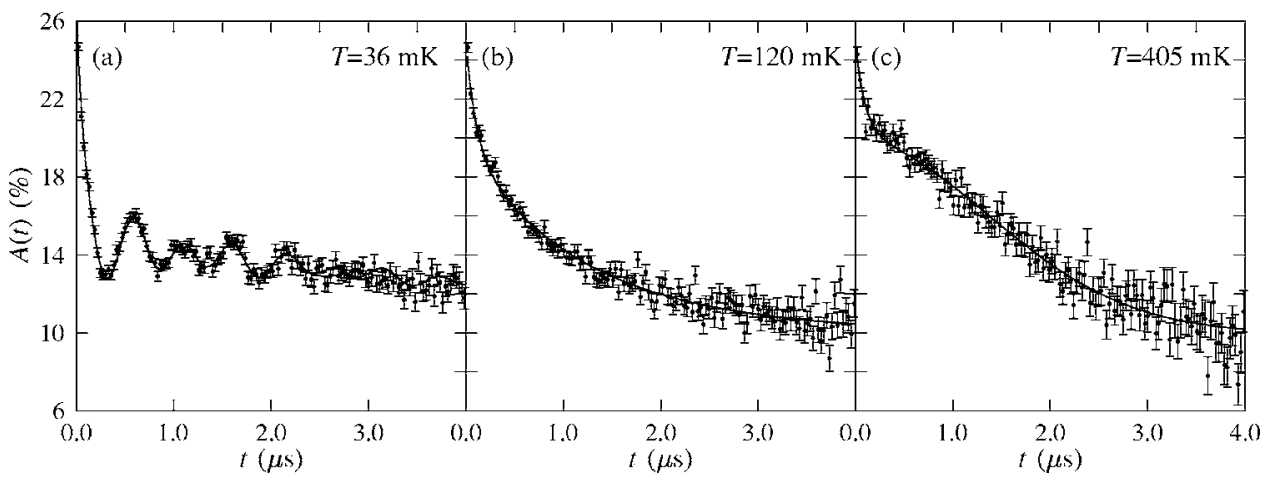

FIG. 1. ZF $\mu^{+} \mathrm{SR}$ spectra for CuPzN at three temperatures. (a) $T=36 \mathrm{mK}$ : Oscillations are observed in the asymmetry, characteristic of long-range magnetic order. A fit is shown in Eq. (2). (b) $T=120 \mathrm{mK}$ : the spectra are described by two components. A fast relaxing signal, probably due to the existence of a radical state, and a larger component, seen to be exponential in form. A fit is shown to Eq. (3). (c) $T$ $=405 \mathrm{mK}$ : The fast relaxing component is still evident although the larger component now shows a Gaussian form. A fit is shown to Eq. (3).

tions are characteristic of a quasistatic local magnetic field at the muon stopping site, which causes a coherent precession of the spins of those muons with a component of their spin polarization perpendicular to this local field (expected to be two-thirds of the total polarization). The frequency of the oscillations is given by $\nu_{i}=\gamma_{\mu} B_{i} / 2 \pi$, where $\gamma_{\mu}$ is the muon gyromagnetic ratio $\left(=2 \pi \times 135.5 \mathrm{MHz} \mathrm{T}^{-1}\right)$ and $B_{i}$ is the average magnitude of the local magnetic field at the $i$ th muon site. Any fluctuation in magnitude of these local fields will result in a relaxation of the oscillating signal, described by relaxation rates $\lambda_{i}$. The presence of oscillations at low temperatures in $\mathrm{CuPzN}$ provides convincing evidence that this material is magnetically ordered below $110 \mathrm{mK}$.

Two separate frequencies were identified in the low temperature spectra, corresponding to two magnetically inequivalent muon stopping sites in the material. We note here that two muon sites have been observed in other copper pyrazine-based compounds. ${ }^{10}$ The precession frequencies, which are proportional to the internal magnetic field as experienced by the muon, may be viewed as an effective order parameter for these systems. In order to extract the temperature dependence of the frequencies, the low temperature data were fitted to the function

$$
\begin{aligned}
A(t)= & A_{1} \exp \left(-\lambda_{1} t\right) \cos \left(2 \pi \nu_{1} t+\phi_{1}\right) \\
& +A_{2} \exp \left(-\lambda_{2} t\right) \cos \left(2 \pi \nu_{2} t+\phi_{2}\right)+A_{3} \exp (-\Lambda t) \\
& +A_{\mathrm{bg}} \exp \left(-\lambda_{\mathrm{bg}} t\right)
\end{aligned}
$$

where $A_{3} \exp (-\Lambda t)$ accounts for the contribution from those muons with a spin component parallel to the local magnetic field. The term $A_{\mathrm{bg}} \exp \left(-\lambda_{\mathrm{bg}} t\right)$ reflects the small signal from those muons that stop in the silver sample holder or cryostat tail (with $\lambda_{\text {bg }} \ll \Lambda$ ).

Across the measured temperature range, the two frequencies were found to be in the proportions $\nu_{1}: \nu_{2}=1: 0.65$ while the relaxation rates $\lambda_{i}$ were found to be in the ratio $\lambda_{1}: \lambda_{2}$ $=1: 0.75$. These quantities were fixed in these ratios during the fitting procedure. The amplitudes and phases best describing the data are given in Ref. 11 . We note that the ratio of longitudinal to transverse components is in excess of the expected value of $A_{3} /\left(A_{1}+A_{2}\right)=1 / 2$ (see below). Nonzero phases were also required to fit the data as observed in previous studies of $\mathrm{Cu}$-based chain compounds of this sort. ${ }^{10}$ The magnitudes of the frequencies were fitted as a function of temperature with other parameters in Eq. (2) fixed. ${ }^{11}$ The resulting temperature evolution of the precession frequencies is shown in Fig. 2(a).

From fits of $\nu_{i}$ to the form $\nu_{i}(T)=\nu_{i}(0)\left(1-T / T_{\mathrm{N}}\right)^{\beta}$ close to $T_{\mathrm{N}}$, we estimate $T_{\mathrm{N}}=107(1) \mathrm{mK}$, yielding $\left|k_{\mathrm{B}} T_{\mathrm{N}} / J\right|$ $=0.0103(1)$. The parameter $\beta$ is highly sensitive to the chosen value of $T_{\mathrm{N}}$ due to the difficulty of obtaining data sufficiently close to the transition. Using $T_{\mathrm{N}}=107(1) \mathrm{mK}$, we es-

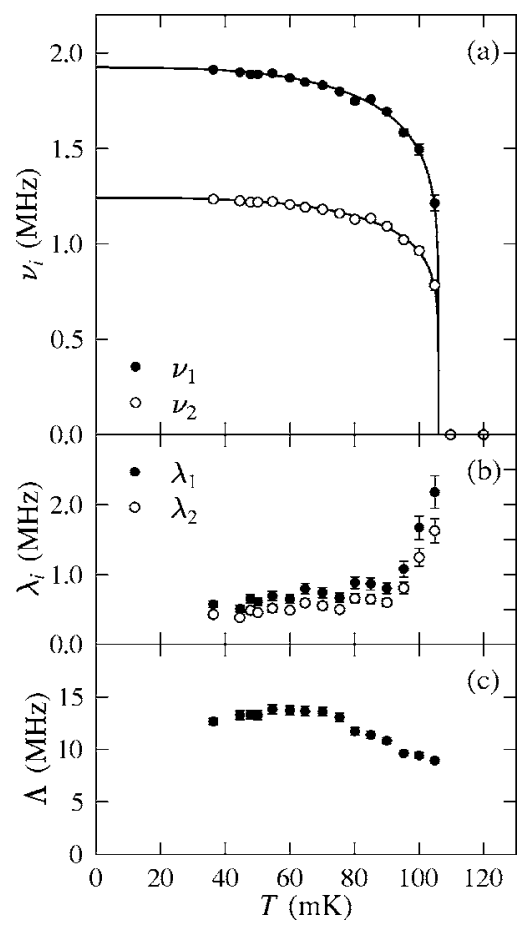

FIG. 2. Temperature evolution of the variable parameters in Eq. (2) for $T \leqslant 140 \mathrm{mK}$. (a) Muon precession frequencies $\nu_{i}$ with fits shown to the expression $\nu_{i}(T)=\nu_{i}(0)\left[1-\left(T / T_{\mathrm{N}}\right)^{\alpha}\right]^{\beta}$. (b) Transverse relaxation rates $\lambda_{i}$ are seen to increase as the magnetic transition is approached from below. (c) The longitudinal relaxation rate $\Lambda$, which decreases as the transition is approached from below. 
timate $\beta=0.18(5)$, which is consistent with low-dimensional (i.e., 2D Ising or $2 \mathrm{D} X Y)$ behavior. Fits to $\nu_{i}(T)=\nu_{i}(0)[1$ $\left.-\left(T / T_{\mathrm{N}}\right)^{\alpha}\right]^{\beta}$ with $\alpha=3$ yield $\nu_{1}(0)=1.922(4) \mathrm{MHz}$ and $\nu_{2}(0)=1.257(3) \mathrm{MHz}$ corresponding to local magnetic fields at the two muon sites of $B_{1}=141.8(3) \mathrm{G}$ and $B_{2}=92.7(2) \mathrm{G}$.

Further evidence for a magnetic transition is seen in the transverse relaxation rates $\lambda_{i}$, which increase as the $T_{\mathrm{N}}$ is approached from below [Fig. 2(b)], as would be expected for critical slowing of fluctuations near a magnetic transition. The longitudinal relaxation rate $\Lambda$ decreases as the transition is approached from below [Fig. 2(c)], suggesting that this quantity is dominated by the magnitude of the local magnetic field at the muon site.

At temperatures just above $T_{\mathrm{N}}$ the measured spectra are best described by two components [Fig. 1(b)]. One is a fast relaxing contribution, with an amplitude $\sim 5 \%$, likely to arise due to a fraction of the muons that initially form radical states. This component will also exist in the magnetically ordered regime but is not resolvable from the longitudinal relaxation and partly explains why the value of $A_{3}$ is greater than that expected. The second component observed in this regime is an exponential functional form, characteristic of fast dynamics in the local magnetic field at the muon sites. ${ }^{12}$ At higher temperatures still $(T>400 \mathrm{mK})$, this second component in the spectra appears Gaussian in form [Fig. 1(c)], characteristic of the early time behavior of a Kubo Toyabe function ${ }^{12}$ due to the magnetic field distribution of a static, disordered system with slow dynamics preventing the characteristic recovery of the polarization at late times. It is likely that at these elevated temperatures, the electronic moments are fluctuating too fast compared to the muon time scale and are therefore motionally narrowed from the spectra. Instead the muon ensemble is depolarized by the magnetic field distribution at the muon site due to the quasistatic nuclear moments of the surrounding nuclei. This interpretation is supported by the observation that applied magnetic fields of magnitude $\geqslant 100 \mathrm{G}$ repolarize the relaxation as expected for a static distribution [repolarization implies $\lim _{t \rightarrow \infty} A(t)$ $=A(0)]$. The measured data for $T>T_{\mathrm{N}}$ were therefore fitted to

$$
A(t)=A_{4} \exp \left(-\lambda_{4} t\right)+A_{5} \exp \left(-\lambda_{5} t\right)^{\delta}+A_{\mathrm{bg}}
$$

where the stretching parameter $\delta$ describes the passage from exponential to Gaussian behavior. The results of this analysis are shown in Fig. 3(a). The increase in the relaxation rate $\lambda_{5}$ as the proposed transition temperature is approached from above lends further weight to the case for the existence of a magnetic transition.

In order to further investigate the high temperature behavior of the material measurements were made in an applied longitudinal magnetic field (LF) $B_{\mathrm{L}}$ using a ${ }^{4} \mathrm{He}$ cryostat at the ISIS facility. The pulsed structure of the muon source at ISIS has the advantage of allowing the observation of the muon polarization at longer times than at a continuous source such as the $\mathrm{S} \mu \mathrm{S}$. This enables us to better characterize the data with the Kubo Toyabe function generalized for LFs. ${ }^{12}$ The loss of time resolution at a pulsed source causes the fast relaxing component described above to be
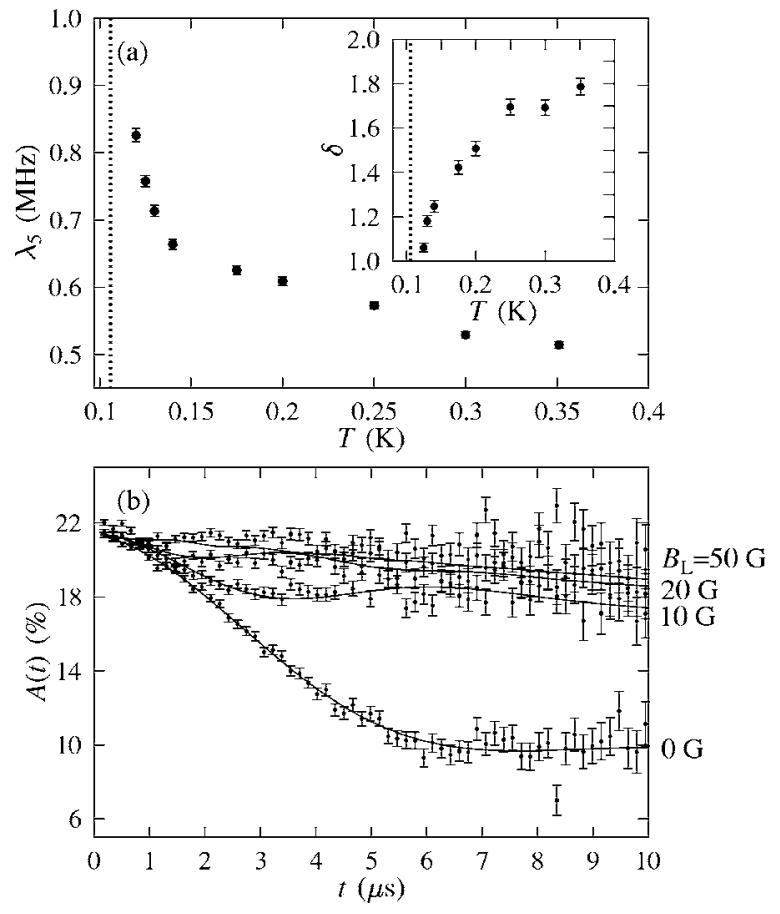

FIG. 3. (a) Results of fitting data for $T>110 \mathrm{mK}$ to Eq. (3). (Dashed lines show $T=T_{\mathrm{N}}$.) Inset: The parameter $\delta$ changing from $\delta=1$ (exponential relaxation) to $\delta=2$ (Gaussian relaxation) as the dynamics move out of the muon time window with increasing temperature. Main panel: The relaxation rate $\lambda_{5}$ increases as the transition is approached from above. (b) LF $\mu^{+} \mathrm{SR}$ spectra made at $T$ $=2 \mathrm{~K}$ in several applied magnetic fields $B_{\mathrm{L}}$. Fits are shown to the LFKT function with $\Delta$ fixed at $0.271 \mathrm{MHz}$ for all fields.

missing from the signal entirely. The results of these measurements, made at $T=2 \mathrm{~K}$, are shown in Fig. 3(b). The spectra are found to be approximately temperature independent in the range $2 \leqslant T \leqslant 50 \mathrm{~K}$. The application of a field repolarizes the slowly relaxing component of asymmetry for $B<100 \mathrm{G}$ consistent with the interpretation that the relaxation at these temperatures is due to the disordered, quasistatic nuclear magnetic moments. The measured ZF and LF spectra for both materials at $2 \mathrm{~K}$ are well described by the longitudinal field Kubo-Toyabe (LFKT) function describing the relaxation due to an ensemble of disordered magnetic moments, although the LFKT function must be multiplied by an exponential function with a constant relaxation rate to account for the weak relaxation due to dynamic fluctuations. ${ }^{13}$ This treatment has only one free parameter $\Delta=\gamma_{\mu} \sqrt{\left\langle B_{\text {loc }}^{2}\right\rangle}$, where $\left\langle B_{\text {loc }}^{2}\right\rangle$ is the second moment of the average local magnetic field distribution at the muon sites in the ZF. This analysis yields a value of $\Delta=0.271 \mathrm{MHz}$ for $\mathrm{CuPzN}$.

An efficacious method for estimating the interchain coupling $J^{\prime}$ in LCHAFMs has recently been developed based on a modified random phase approximation and modeled with classical and quantum Monte Carlo simulations. ${ }^{14}$ This approach leads to an empirical formula for the interchain coupling given by 
TABLE I. Parameters for Cu-chain compounds $\mathrm{Sr}_{2} \mathrm{CuO}_{3}$, (Ref. [16]) $\mathrm{KCuF}_{3}$, (Ref. [17]) $\mathrm{CPC}\left(=\mathrm{Cu}(\text { py })_{2} \mathrm{Cl}_{2}\right)$ (Ref. [15]) and $\mathrm{CuPzN}$ (Ref. 4 and this work) with values of $\left|J^{\prime} / J\right|$ estimated from Eq. (4).

\begin{tabular}{lcccc}
\hline \hline & $|J| / k_{\mathrm{B}}(\mathrm{K})$ & $T_{\mathrm{N}}(\mathrm{K})$ & $\left|k_{\mathrm{B}} T_{\mathrm{N}} / J\right|$ & $\left|J^{\prime} / J\right|$ \\
\hline $\mathrm{Sr}_{2} \mathrm{CuO}_{3}$ & 2200 & 5.4 & $2.5 \times 10^{-3}$ & $9.3 \times 10^{-4}$ \\
$\mathrm{KCuF}_{3}$ & 406 & 39 & $9.6 \times 10^{-2}$ & $5.2 \times 10^{-2}$ \\
$\mathrm{CPC}$ & 26 & 1.1 & $4.2 \times 10^{-2}$ & $2.1 \times 10^{-2}$ \\
$\mathrm{CuPzN}$ & 10.3 & 0.107 & $1.0 \times 10^{-2}$ & $4.4 \times 10^{-3}$ \\
\hline \hline
\end{tabular}

$$
\left|J^{\prime}\right| / k_{\mathrm{B}}=\frac{T_{\mathrm{N}}}{4 c \sqrt{\ln \left(\frac{\lambda|J|}{k_{\mathrm{B}} T_{\mathrm{N}}}\right)+\frac{1}{2} \ln \ln \left(\frac{\lambda|J|}{k_{\mathrm{B}} T_{\mathrm{N}}}\right)}},
$$

with $\lambda=2.6$ and $c=0.233$. Substituting our value of $T_{\mathrm{N}}$ $=107(1) \mathrm{mK}$ and using $J / k_{\mathrm{B}}=-10.3(1) \mathrm{K}$, yields $\left|J^{\prime}\right| / k_{\mathrm{B}}$ $\simeq 0.046 \mathrm{~K}$ and $\left|J^{\prime} / J\right|=4.4 \times 10^{-3}$. The parameters describing four $\mathrm{Cu}$-chain compounds are presented in Table I. Comparing values of $\left|J^{\prime} / J\right|$ shows that $\mathrm{CuPzN}$ is approximately five times better isolated than copper bispyridine dichloride $\left(\mathrm{Cu}(\mathrm{py})_{2} \mathrm{Cl}_{2}\right.$ or $\left.\mathrm{CPC}\right),{ }^{15}$ while $\left|J^{\prime} / J\right|$ for $\mathrm{CuPzN}$ is around one quarter of that for the best isolated LCHAFM, $\mathrm{Sr}_{2} \mathrm{CuO}_{3}$ (although this material has a QCP at a field around 200 times larger than that for $\mathrm{CuPzN}) .{ }^{16}$

The failure of specific heat studies ${ }^{8}$ to detect an anomaly due to the onset of a 3D LRO in CuPzN down to $70 \mathrm{mK}$ could be due to various factors, including the strain induced by vacuum grease or insufficient data points. However, it may be that the small value of $\left|J^{\prime} / J\right|$ makes specific heat measurements unsuitable for detecting the transition. Investigations using a stochastic series quantum Monte Carlo method $^{19}$ show that the height of the expected peak in the specific heat at $T_{\mathrm{N}}$ decreases with decreasing $\left|J^{\prime}\right|$ becoming approximately proportional to $\left|J^{\prime} / J\right|$ below $\left|J^{\prime} / J\right|=0.2$. Thus when $\left|J^{\prime} / J\right|$ is very small the entropy change at $T_{\mathrm{N}}$ is so small that it makes an undetectable contribution to the specific heat.

In conclusion we have demonstrated the existence of long range magnetic order in the LCHAFM material $\mathrm{CuPzN}$ with $T_{\mathrm{N}}=107(1) \mathrm{mK}$. This leads to an estimate of the interchain coupling of $\left|J^{\prime}\right| / k_{\mathrm{B}} \simeq 0.046 \mathrm{~K}$. For many $1 \mathrm{D}$ systems small values of $\left|J^{\prime} / J\right|$ prevent the detection of 3D LRO with specific heat, and reduced ordered moments make the use of other techniques problematical (coupled chain mean-field theory ${ }^{18}$ then predicts an ordered moment size of $\sim 0.05 \mu_{\mathrm{B}}$ for $\mathrm{CuPzN}$ ). Thus we expect that muons may provide a particularly valuable tool for future magnetic measurements of the key parameters of highly anisotropic low-dimensional systems.

Parts of this work were carried out at the $\mathrm{S} \mu \mathrm{S}$ and ISIS. We thank W. Hayes for useful discussions and EPSRC for financial support. T.L acknowledges support from the European Commission, Contract No. RII3-CT-2003-505925
*Electronic address: t.lancaster1@physics.ox.ac.uk

${ }^{1}$ G. Müller, H. Thomas, H. Beck, and J. C. Bonner, Phys. Rev. B 24, 1429 (1981); M. Karbach, G. Müller, A. H. Bougourzi, A. Fledderjohann, and K. H. Mütter, Phys. Rev. B 55, 12510 (1997).

${ }^{2}$ S. Sachdev, Quantum Phase Transitions (Cambridge University Press, Cambridge, 2000).

${ }^{3}$ N. M. Bogoliubov, A. G. Izergin, and V. E. Korepin, Nucl. Phys. B 275, 687 (1986); A. Fledderjohann, C. Gerhardt, K. H. Mütter, A. Schmitt, and M. Karbach, Phys. Rev. B 54, 7168 (1996).

${ }^{4}$ P. R. Hammar et al., Phys. Rev. B 59, 1008 (1999).

${ }^{5}$ J. Villain and J. M. Loveluck, J. Phys. (France) Lett. 38, L77 (1977)

${ }^{6}$ M. B. Stone, D. H. Reich, C. Broholm, K. Lefmann, C. Rischel, C. P. Landee, and M. M. Turnbull, Phys. Rev. Lett. 91, 037205 (2003).

${ }^{7}$ K. Lefmann and C. Rischel, Phys. Rev. B 54, 6340 (1996); M. Karbach and G. Müller, Phys. Rev. B 62, 14871 (2000); M. Karbach, D. Biegel, and G. Müller, Phys. Rev. B 66, 054405 (2002).

${ }^{8}$ G. Mennenga, L. J. de Jongh, W. J. Huiskamp, and J. Reedijk, J. Magn. Magn. Mater. 44, 89 (1984).
${ }^{9}$ S. J. Blundell, Contemp. Phys. 40, 175 (1999).

${ }^{10}$ T. Lancaster et al., J. Phys.: Condens. Matter 16, S4563 (2004).

${ }^{11}$ The fixed fitting parameters for Eq. (2) best describing the data measured below $T=110 \mathrm{mK}$ were found to be $A_{1}=1.56 \%, \phi_{1}$ $=-37.8^{\circ}, A_{2}=0.63 \%, \phi_{2}=-73.5^{\circ}$, and $A_{3}=10.42 \%$.

${ }^{12}$ R. S. Hayano, Y. J. Uemura, J. Imazato, N. Nishida, T. Yamazaki, and R. Kubo, Phys. Rev. B 20, 850 (1979).

${ }^{13}$ The relaxation function multiplied by the LFKT function may be used to extract information on spin-wave dynamics in these materials; F. L. Pratt et al. (unpublished).

${ }^{14}$ C. Yasuda, S. Todo, K. Hukushima, F. Alet, M. Keller, M. Troyer, and H. Takayama, Phys. Rev. Lett. 94, 217201 (2005).

${ }^{15}$ W. Duffy Jr., J. E. Venneman, D. L. Strandburg, and P. M. Richards, Phys. Rev. B 9, 2220 (1974).

${ }^{16}$ N. Motoyama, H. Eisaki, and S. Uchida, Phys. Rev. Lett. 76, 3212 (1996); K. M. Kojima et al., Phys. Rev. Lett. 78, 1787 (1997).

${ }^{17}$ S. K. Satija, J. D. Axe, G. Shirane, H. Yoshizawa, and K. Hirakawa, Phys. Rev. B 21, 2001 (1980).

${ }^{18}$ H. J. Schulz, Phys. Rev. Lett. 77, 2790 (1996).

${ }^{19}$ P. Sengupta, A. W. Sandvik, and R. R. P. Singh, Phys. Rev. B 68, 094423 (2003). 